\title{
The Essential Role of Cytokinin Signaling in Root Apical Meristem Formation during Somatic Embryogenesis
}

\author{
Lei Wang and Kang Chong * \\ Key Laboratory of Plant Molecular Physiology, Institute of Botany, Chinese Academy of Sciences, Beijing, China
}

Keywords: somatic embryogenesis, hypophysis, shoot-root axis, auxin gradients, cytokinin response, RAM establishment

\section{A commentary on}

Establishment of embryonic shoot-root axis is involved in auxin and cytokinin response during Arabidopsis somatic embryogenesis

by Su, Y. H., Liu, Y. B., Bai, B., and Zhang, X. S. (2015). Front. Plant Sci. 5:792. doi: $10.3389 / \mathrm{fpls} .2014 .00792$

Plant tissue culture and subsequent organogenesis have been regarded as part of the scientific roots of modern plant biotechnology (Sussex, 2008). Establishments of shoot apical meristem (SAM) and root apical meristem (RAM) are critical steps for somatic embryogenesis (Scheres, 2007). WUSCHEL (WUS), a homeodomain protein, controls stem cell differentiation and maintenance and its ectopic expression facilitated the transition from vegetative growth to embryogenesis in Arabidopsis (Zuo et al., 2002). Gain-of-function of WUS mutant can trigger cell pluripotence and re-establishing new meristems in the cortex, which further reinforced the essential role of WUS in the initiation and maintenance of meristem in planta (Xu et al., 2005). Nevertheless, the underlying mechanisms of how somatic cell maintains plasticity and triggers cellular switch during the sex-free embryogenesis are still elusive, which is one of the top 25 profound as-yet-unanswered scientific questions (Vogel, 2005). During early somatic embryogenesis, while auxin signaling cascade induces the expression of WUS (Su et al., 2011), little is known about the underlying mechanisms for the RAM establishment.

The quiescent center (QC) is formed from apical descendant cell after asymmetric division of the hypophysis and essential in maintaining the identity of root stem cells (Möller and Weijers, 2009; Peris et al., 2010). WUSCHEL-RELATED HOMEOBOX 5 (WOX5) plays an analogous role in the QC of the RAM as WUS in organizing center (OC) of the SAM. PLETHORA1 (PLT1) and PLT2, two AP2-type transcription factors, are required to specify and maintain the stem-cell identity in the RAM (Galinha et al., 2007). In addition, transcription factors SCARECROW (SCR) and SHORTROOT (SHR) are necessary to maintain stem cell activity in the RAM (Sabatini et al., 2003). In this study, Dr Xian Sheng Zhang's lab exploited the marker lines of the above genes to investigate the specification and initiation of RAM during nongametic somatic embryogenesis. They detected almost simultaneously induced WOX5 and PLT2 expression in some regions of embryonic callus only $24 \mathrm{~h}$ after transferred onto 2,4-D free somatic embryo-inducing medium (SEIM), while the expression of SCR was detected later in 24-36 h. Further, the spatiotemporal expression patterns of WUS and WOX 5 were examined by tagged with distinct fluorescence protein markers. Nearly identical expression patterns of them were found after $24 \mathrm{~h}$ induction. The WOX5 signals were then detected below the regions of WUS signals, suggesting that an early shoot-root 
axis had been established at this time. Su and colleagues also employed inducible antisense WOX5 transgenic lines and plt2 mutant to investigate their roles for embryonic root formation. Strikingly, significantly decreased number of somatic embryos (SEs) and severely defected SEs were produced from embryonic callus of WOX5 antisense lines and plt2-1 mutant respectively, confirmed that WOX5 and PLT2 are required for somatic embryogenesis, and further indicated the role of WOX5 in the RAM is comparable to that of WUS in the SAM.

In zygotic embryos, auxin and cytokinin display inverse correlation in specifying the root stem cell niche (Müller and Sheen, 2008). In this scenario, auxin signaling antagonizes cytokinin responses during zygotic embryogenesis by directly inducing the transcription of ARABIDOPSIS RESPONSE REGULATOR (ARR) 7 and ARR15, the two critical A-type ARR transcription factors to repress cytokinin signaling (Figure 1), which is essential in establishing the root stem cell niche (Müller and Sheen, 2008). Intriguingly, ARR7 and ARR15 expression is stimulated during root specification, but repressed by the auxin signal in the SAM of the embryo (Zhao et al., 2010). However, the interaction of auxin and cytokinin in the establishment of root stem cell niche in somatic embryogenesis is less clear. $\mathrm{Su}$ and her colleagues further traced auxin response signals by utilizing DR5rev promoter driven VENUS or GFP expression and found that its spatiotemporal expression patterns were overlapped with neither WOX5:GFP nor PLT2:RFP. Instead, they showed that $A R R 7$ and $A R R 15$, which also can be induced by cytokinin (Werner and Schmülling, 2009), displayed overlapping expression patterns with WOX5 rather than WUS. This remarkable evidence implied that cytokinin, but not auxin, is essential for initiation of the RAM of SE (Figure 1). This was further supported by the observation of defective SEs in the overexpressing lines of $A R R 7$ and $A R R 15$, and double mutants of $a h k 2 a h k 4$ and $a h k 3 a h k 4$. The authors finally demonstrated that cytokinin signaling strictly confined WOX5 expression to the site of subsequently embryonic root meristem by in situ hybridization (Su et al., 2015).

The establishment of the shoot-root axis is a crucial process for both zygotic and somatic embryogenesis. Despite the processes of the two kinds of embryogenesis are quite similar from globular to torpedo stage (Zimmerman, 1993), the underlying mechanisms seem to be different in at least two aspects. (1) The spatiotemporal expression patterns of WUS and WOX genes are distinct. In zygotic embryogenesis, WUS and WOX5 are sequentially expressed with roughly $12 \mathrm{~h}$ interval. While during somatic embryogenesis they are simultaneously expressed in nearly overlapping regions of embryonic callus, indicating that SAM and RAM are almost initiated at the overlapping regional domains of callus and formed the apicalbasal axis at early stages of somatic embryogenesis. (2) The roles of auxin gradient response and cytokinin signaling show discrepancy in zygotic and somatic embryogenesis (Figure 1). In planta, the proper establishment of auxin gradient response is essential for correct spatiotemporal cytokinin signaling, which causes the lower expression of $A R R 7 / A R R 15$ in the upper lens-shaped cell but higher expression in the lower large basal cell (Müller and Sheen, 2008). Cytokinin signaling plays a critical role during root stem cell niche formation in zygotic embryogenesis as chemical induced double mutant arr7arr15 produced the defective RAM system. In here, Su and her colleagues' compelling results demonstrated that cytokinin signaling is also essential for RAM initiation in SE. Collectively, their results provide novel insights into the roles of cytokinin and auxin in the formation of shoot-root axis during somatic embryogenesis, and contribute to the ongoing investigation of the mechanisms of somatic embryogenesis. These knowledges are invaluable in propagating the endangered

\section{In zygotic embryogenesis}

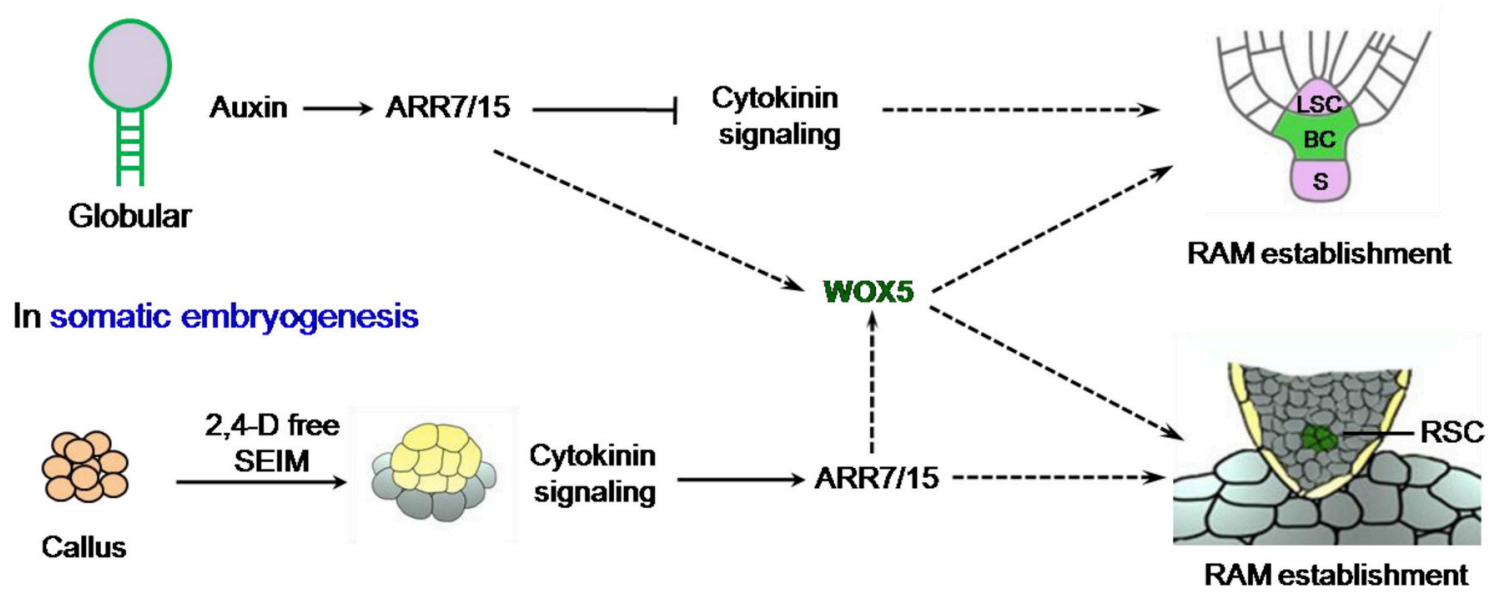

FIGURE 1 | Comparison of the roles of auxin and cytokinin during RAM specification in zygotic and somatic embryos. The cells with green color represent established RAM niche in zygotic embryogenesis (ZE) and somatic embryogenesis (SE) respectively. The dashed lines stand for proposed molecular link but need to be further corroborated. S, Suspensor; LSC, Lens-Shaped Cell; BC, Basal Cell lineage; RSC, root stem cells of SE. 
and indispensable plant species and in molecular breeding of crops through callus-based plant transformation in the near future.

\section{AUTHOR CONTRIBUTIONS}

LW and KC conceived the research and wrote the manuscript.

\section{REFERENCES}

Galinha, C., Hofhuis, H., Luijten, M., Willemsen, V., Blilou, I., Heidstra, R., et al. (2007). PLETHORA proteins as dose-dependent master regulators of Arabidopsis root development. Nature 449, 1053-1057. doi: 10.1038 /nature 06206

Möller, B., and Weijers, D. (2009). Auxin control of embryo patterning. Cold Spring Harb. Perspect. Biol. 1:a001545. doi: 10.1101/cshperspect.a001545

Müller, B., and Sheen, J. (2008). Cytokinin and auxin interaction in root stemcell specification during early embryogenesis. Nature 453, 1094-1097. doi: 10.1038/nature06943

Peris, C. I., Rademacher, E. H., and Weijers, D. (2010). Green beginnings-pattern formation in the early plant embryo. Curr. Top. Dev. Biol. 91, 1-27. doi: 10.1016/S0070-2153(10)91001-6

Sabatini, S., Heidstra, R., Wildwater, M., and Scheres, B. (2003). SCARECROW is involved in positioning the stem cell niche in the Arabidopsis root meristem. Genes Dev. 17, 354-358. doi: 10.1101/gad.252503

Scheres, B. (2007). Stem-cell niches: nursery rhymes across kingdoms. Nat. Rev. Mol. Cell Biol. 8, 345-354. doi: 10.1038/nrm2164

Su, Y. H., Liu, Y. B., and Zhang, X. S. (2011). Auxin-cytokin in interaction regulates meristem development. Mol. Plant. 4, 616-625. doi: 10.1093/mp/ssr007

Su, Y. H., Li,Y. B., Bai, B., and Zhang, X. S. (2015). Establishment of embryonic shoot-root axis is involved in auxin and cytokinin response during Arabidopsis somatic embryogenesis. Front. Plant Sci. 5:792. doi: 10.3389/fpls. 2014.00792

Sussex, I. M. (2008). The scientific roots of modern plant biotechnology. Plant Cell 20, 1189-1198. doi: 10.1105/tpc.108.058735

\section{ACKNOWLEDGMENTS}

We are grateful for Dr. Xiansheng Zhang's critical reading and valuable comments on this manuscript. The authors would like to acknowledge funding from National Natural Science Foundation of China (No. 31570292) for LW and (No. 30821007) for KC, and from the Major State Basic Research Program of China (2013CBA01403) for KC.

Vogel, G. (2005). How does a single somatic cell become a whole plant? Science 309:86 doi: $10.1126 /$ science.309.5731.86

Werner, T., and Schmülling, T. (2009). Cytokinin action in plant development. Curr. Opin. Plant Biol. 12, 527-538. doi: 10.1016/j.pbi.2009.07.002

Xu, Y. Y., Wang, X. M., Li, J., Li, J. H., Wu, J. S., Walker, J. C., et al. (2005). Activation of the WUS gene induces ectopic initiation of floral meristems on mature stem surface in Arabidopsis thaliana. Plant Mol. Biol. 57, 773-784. doi: 10.1007/s11103-005-0952-9

Zhao, Z., Andersen, S. U., Ljung, K., Dolezal, K., Miotk, A., Schultheiss, S. J., et al. (2010). Hormonal control of the shoot stem cell niche. Nature 465, 1089-1092. doi: $10.1038 /$ nature09126

Zimmerman, J. L. (1993). Somatic embryogenesis: a model for early development in higher plants. Plant Cell 5, 1411-1423. doi: 10.1105/tpc.5.10.1411

Zuo, J., Niu, Q.-W., Frugis, G., and Chua, N.-H. (2002). The WUSCHEL gene promotes vegetative-to-embryonic transition in Arabidopsis. Plant J. 30, 349-359. doi: 10.1046/j.1365-313X.2002.01289.x

Conflict of Interest Statement: The authors declare that the research was conducted in the absence of any commercial or financial relationships that could be construed as a potential conflict of interest.

Copyright (c) 2016 Wang and Chong. This is an open-access article distributed under the terms of the Creative Commons Attribution License (CC BY). The use, distribution or reproduction in other forums is permitted, provided the original author(s) or licensor are credited and that the original publication in this journal is cited, in accordance with accepted academic practice. No use, distribution or reproduction is permitted which does not comply with these terms. 